\title{
Social force model for pedestrian dynamics
}

\author{
Dirk Helbing and Péter Molnár \\ II. Institute of Theoretical Physics, University of Stuttgart, 70550 Stuttgart, Germany
}

\begin{abstract}
It is suggested that the motion of pedestrians can be described as if they would be subject to 'social forces'. These 'forces' are not directly exerted by the pedestrians' personal environment, but they are a measure for the internal motivations of the individuals to perform certain actions (movements). The corresponding force concept is discussed in more detail and can be also applied to the description of other behaviors.

In the presented model of pedestrian behavior several force terms are essential: First, a term describing the acceleration towards the desired velocity of motion. Second, terms reflecting that a pedestrian keeps a certain distance to other pedestrians and borders. Third, a term modeling attractive effects. The resulting equations of motion are nonlinearly coupled LANGEVIN equations. Computer simulations of crowds of interacting pedestrians show that the social force model is capable of describing the self-organization of several observed collective effects of pedestrian behavior very realistically.
\end{abstract}

Typeset using REVTEX 


\section{INTRODUCTION}

During the last two decades models of pedestrian behavior have found notable interest for several reasons. First, there are some striking analogies with gases [1, 3] and fluids [1, 2, 4, 4 . Second, all model quantities like places $\vec{r}_{\alpha}$ and velocities $\vec{v}_{\alpha}$ of pedestrians $\alpha$ are measurable and, therefore, comparable with empirical data. Third, there already exists a considerable amount of data material like flow measurements or (video) films [6 8]. Fourth, pedestrian models can provide valuable tools for designing and planning pedestrian areas, subway or railroad stations, big buildings, shopping malls, etc. [9 [1].

In the 1970s HENDERSON [4,5] has compared measurements of pedestrian flows with NAviER-StOKES equations with considerable success. His phenomenological fluid-dynamic approach has been improved and mathematically founded by HELBING in the 1990s on the basis of a pedestrian specific gaskinetic (Boltzmann-like) model [1,2]. This model is closely

related to some gaskinetic [12,13] and fluid-dynamic [14 16] traffic models. Recently, much attention has been attracted by 'microscopic' approaches for vehicular traffic [17 20. In the following, we will introduce a social force model for pedestrian motion. First ideas of this 'microscopic' modeling concept were formulated in [2,21]. A short overview of 'microscopic', 'mesoscopic' and 'macroscopic' descriptions of pedestrian behavior and their interrelations is given in Ref. [22].

\section{THE SOCIAL FORCE CONCEPT}

Many people have the feeling that human behavior is 'chaotic' or at least very irregular and not predictable. This is probably true for behaviors that are found in complex situations. However, at least for relatively simple situations stochastic behavioral models may be developed if one restricts to the description of behavioral probabilities that can be found in a huge population (resp. group) of individuals (see [3,23,24]). This idea has been followed by the gaskinetic pedestrian model $11-3]$. 
Another approach for modeling behavioral changes has been suggested by LEWIN [25]. According to his idea behavioral changes are guided by socalled social fields resp. social forces. In the following we will examine if this idea could be applied to the description of pedestrian behavior.

Figure 1 illustrates a scheme of the processes that lead to behavioral changes. According to this, a sensory stimulus causes a behavioral reaction that depends on the personal aims and is chosen from a set of behavioral alternatives with the objective of utility maximization.

Table 1 suggests a classification of stimuli into simple or standard situations that are well predictable, and complex or new situations that may be modelled with probabilistic models. However, since a pedestrian is used to the situations he/she is normally confronted with, his/her reaction is usually rather automatic, and determined by his/her experience of which reaction will be the best. It is therefore possible to put the rules of pedestrian behavior into an equation of motion. According to this equation the systematic temporal changes $d \vec{w}_{\alpha} / d t$ of the prefered velocity $\vec{w}_{\alpha}(t)$ of a pedestrian $\alpha$ are described by a vectorial quantity $\vec{F}_{\alpha}(t)$ that can be interpreted as social force. Clearly, this force must represent the effect of the environment (e.g. other pedestrians or borders) on the behavior of the described pedestrian. However, the social force is not excerted by the environment on a pedestrian's body. It is rather a quantity that describes the concrete motivation to act. In the case of pedestrian behavior this motivation evokes the physical production of an acceleration or deceleration force as reaction to the perceived information that he/she obtains about his/her environment (see figure 1). In summary, one can say that a pedestrian acts as if he/she would be subject to external forces. This idea has been mathematically founded in Ref. [26].

\section{FORMULATION OF THE SOCIAL FORCE MODEL}

In the following the main effects that determine the motion of a pedestrian $\alpha$ will be introduced:

1. He/She wants to reach a certain destination $\vec{r}_{\alpha}^{0}$ as comfortable as possible. Therefore, 
he/she normally takes a way without detours, i.e., the shortest possible way. This way will usually have the shape of a polygon with edges $\vec{r}_{\alpha}^{1}, \ldots, \vec{r}_{\alpha}^{n}:=\vec{r}_{\alpha}^{0}$. If $\vec{r}_{\alpha}^{k}$ is the next edge of this polygon to reach, his/her desired direction $\vec{e}_{\alpha}(t)$ of motion will be

$$
\vec{e}_{\alpha}(t):=\frac{\vec{r}_{\alpha}^{k}-\vec{r}_{\alpha}(t)}{\left\|\vec{r}_{\alpha}^{k}-\vec{r}_{\alpha}(t)\right\|},
$$

where $\vec{r}_{\alpha}(t)$ denotes the actual position of pedestrian $\alpha$ at time $t$. Exactly speaking, the goals of a pedestrian are usually rather gates or areas than points $\vec{r}_{\alpha}^{k}$. In this case, he/she will at every time $t$ steer for the nearest point $\vec{r}_{\alpha}^{k}(t)$ of the corresponding gate/area.

If a pedestrian's motion is not disturbed, he/she will walk into the desired direction $\vec{e}_{\alpha}(t)$ with a certain desired speed $v_{\alpha}^{0}$. A deviation of the actual velocity $\vec{v}_{\alpha}(t)$ from the desired velocity $\vec{v}_{\alpha}^{0}(t):=v_{\alpha}^{0} \vec{e}_{\alpha}(t)$ due to necessary deceleration processes or avoidance processes leads to a tendency to approach $\vec{v}_{\alpha}^{0}(t)$ again within a certain relaxation time $\tau_{\alpha}$. This can be described by an acceleration term of the form

$$
\vec{F}_{\alpha}^{0}\left(\vec{v}_{\alpha}, v_{\alpha}^{0} \vec{e}_{\alpha}\right):=\frac{1}{\tau_{\alpha}}\left(v_{\alpha}^{0} \vec{e}_{\alpha}-\vec{v}_{\alpha}\right)
$$

2. The motion of a pedestrian $\alpha$ is influenced by other pedestrians. Especially, he/she keeps a certain distance from other pedestrians that depends on the pedestrian density and the desired speed $v_{\alpha}^{0}$. Here, the privat sphere of each pedestrian, which can be interpreted as territorial effect [27], plays an essential role. A pedestrian normally feels increasingly incomfortable the closer he/she gets to a strange person, who may react in an aggressive way. This results in repulsive effects of other pedestrians $\beta$ that can be represented by vectorial quantities

$$
\vec{f}_{\alpha \beta}\left(\vec{r}_{\alpha \beta}\right):=-\nabla_{\vec{r}_{\alpha \beta}} V_{\alpha \beta}\left[b\left(\vec{r}_{\alpha \beta}\right)\right] .
$$

We will assume that the repulsive potential $V_{\alpha \beta}(b)$ is a monotonic decreasing function of $b$ with equipotential lines having the form of an ellipse that is directed into the 
direction of motion. The reason for this is that a pedestrian requires space for the next step which is taken into account by other pedestrians. $b$ denotes the semi-minor axis of the ellipse and is given by

$$
2 b:=\sqrt{\left(\left\|\vec{r}_{\alpha \beta}\right\|+\left\|\vec{r}_{\alpha \beta}-v_{\beta} \Delta t \vec{e}_{\beta}\right\|\right)^{2}-\left(v_{\beta} \Delta t\right)^{2}},
$$

where $\vec{r}_{\alpha \beta}:=\vec{r}_{\alpha}-\vec{r}_{\beta} . s_{\beta}:=v_{\beta} \Delta t$ is of the order of the step width of pedestrian $\beta$. Despite the simplicity of this approach, it describes avoidance maneuvers of pedestrians quite realistically.

A pedestrian also keeps a certain distance from borders of buildings, walls, streets, obstacles, etc. [8]. He/She feels the more incomfortable the closer to a border he/she walks since he/she has to pay more attention to avoid the danger of getting hurt, e.g. by accidentally touching a wall. Therefore, a border $B$ evokes a repulsive effect that can be described by

$$
\vec{F}_{\alpha B}\left(\vec{r}_{\alpha B}\right):=-\nabla_{\vec{r}_{\alpha B}} U_{\alpha B}\left(\left\|\vec{r}_{\alpha B}\right\|\right)
$$

with a repulsive and monotonic decreasing potential $U_{\alpha B}\left(\left\|\vec{r}_{\alpha B}\right\|\right)$. Here, the vector $\vec{r}_{\alpha B}:=\vec{r}_{\alpha}-\vec{r}_{B}^{\alpha}$ has been introduced, where $\vec{r}_{B}^{\alpha}$ denotes the location of that piece of border $B$ that is nearest to pedestrian $\alpha$.

3. Pedestrians are sometimes attracted by other persons (friends, street artists, etc.) or objects (e.g. window displays). These attractive effects $\vec{f}_{\alpha i}$ at places $\vec{r}_{i}$ can be modelled by attractive, monotonic increasing potentials $W_{\alpha i}\left(\left\|\vec{r}_{\alpha i}\right\|, t\right)$ in a similar way like the repulsive effects:

$$
\vec{f}_{\alpha i}\left(\left\|\vec{r}_{\alpha i}\right\|, t\right):=-\nabla_{\vec{r}_{\alpha i}} W_{\alpha i}\left(\left\|\vec{r}_{\alpha i}\right\|, t\right)
$$

$\left(\vec{r}_{\alpha i}:=\vec{r}_{\alpha}-\vec{r}_{i}\right)$. The main difference is that the attractiveness $\left\|\vec{f}_{\alpha i}\right\|$ is normally decreasing with time $t$ since the interest is declining. The attractive effects are, e.g., responsible for the formation of pedestrian groups (that are comparable to molecules). 
However, the formulas above for attractive and repulsive effects only hold for situations that are perceived in the desired direction $\vec{e}_{\alpha}(t)$ of motion. Situations located behind a pedestrian will have a weaker influence $c$ with $0<c<1$. In order to take this effect of perception (i.e. of the effective angle $2 \varphi$ of sight) into account we have to introduce the direction dependent weights

$$
w(\vec{e}, \vec{f}):=\left\{\begin{array}{l}
1 \text { if } \vec{e} \cdot \vec{f} \geq\|\vec{f}\| \cos \varphi \\
c \text { otherwise. }
\end{array}\right.
$$

In summary, the repulsive and attractive effects on a pedestrian's behavior are given by

$$
\begin{aligned}
\vec{F}_{\alpha \beta}\left(\vec{e}_{\alpha}, \vec{r}_{\alpha}-\vec{r}_{\beta}\right) & :=w\left(\vec{e}_{\alpha},-\vec{f}_{\alpha \beta}\right) \vec{f}_{\alpha \beta}\left(\vec{r}_{\alpha}-\vec{r}_{\beta}\right), \\
\vec{F}_{\alpha i}\left(\vec{e}_{\alpha}, \vec{r}_{\alpha}-\vec{r}_{i}, t\right) & :=w\left(\vec{e}_{\alpha}, \vec{f}_{\alpha i}\right) \vec{f}_{\alpha i}\left(\vec{r}_{\alpha}-\vec{r}_{i}, t\right) .
\end{aligned}
$$

We can now set up the equation for a pedestrian's total motivation $\vec{F}_{\alpha}(t)$. Since all the previously mentioned effects influence a pedestrian's decision at the same moment, we will assume that their total effect is given by the sum of all effects, like this is the case for forces. This results in

$$
\begin{aligned}
\vec{F}_{\alpha}(t) & :=\vec{F}_{\alpha}^{0}\left(\vec{v}_{\alpha}, v_{\alpha}^{0} \vec{e}_{\alpha}\right)+\sum_{\beta} \vec{F}_{\alpha \beta}\left(\vec{e}_{\alpha}, \vec{r}_{\alpha}-\vec{r}_{\beta}\right) \\
& +\sum_{B} \vec{F}_{\alpha B}\left(\vec{e}_{\alpha}, \vec{r}_{\alpha}-\vec{r}_{B}^{\alpha}\right)+\sum_{i} \vec{F}_{\alpha i}\left(\vec{e}_{\alpha}, \vec{r}_{\alpha}-\vec{r}_{i}, t\right) .
\end{aligned}
$$

The social force model is now defined by

$$
\frac{d \vec{w}_{\alpha}}{d t}:=\vec{F}_{\alpha}(t)+\text { fluctuations } .
$$

Here, we have added a fluctuation term that takes into account random variations of the behavior. These fluctuations stem, on the one hand, from ambiguous situations in which two or more behavioral alternatives are equivalent (e.g. if the utility of passing an obstacle on the right or left hand side is the same). On the other hand, fluctuations arise from accidental or deliberate deviations from the usual rules of motion.

In order to complete the model of pedestrian dynamics a relation between the actual velocity $\vec{v}_{\alpha}(t)$ and the prefered velocity $\vec{w}_{\alpha}(t)$ must be introduced. Since the actual speed 
is limited by a pedestrian's maximal acceptable speed $v_{\alpha}^{\max }$, we will assume that the realized motion is given by

$$
\frac{d \vec{r}_{\alpha}}{d t}=\vec{v}_{\alpha}(t):=\vec{w}_{\alpha}(t) g\left(\frac{v_{\alpha}^{\max }}{\left\|\vec{w}_{\alpha}\right\|}\right)
$$

with

$$
g\left(\frac{v_{\alpha}^{\max }}{\left\|\vec{w}_{\alpha}\right\|}\right):= \begin{cases}1 & \text { if }\left\|\vec{w}_{\alpha}\right\| \leq v_{\alpha}^{\max } \\ v_{\alpha}^{\max } /\left\|\vec{w}_{\alpha}\right\| & \text { otherwise. }\end{cases}
$$

Note that the pedestrian model (10), (11) has the form of nonlinearly coupled LANGEVIN equations. A simplified version of it can be extended to an active walker model [28 30] for the self-organization of systems of ways [31]. This is related to the mathematical description of trunk trail formation by ants [32].

\section{COMPUTER SIMULATIONS}

The model of pedestrian dynamics developed in Section III has been simulated on a computer for a large number of interacting pedestrians confronted with different situations. Despite the fact that the the proposed model is very simple it describes a lot of observed phenomena very realistically. In the following, two examples will be presented showing the self-organization of collective phenomena of pedestrian behavior.

The simulations assumed that the desired speeds $v^{0}$ are GAUSSIAN distributed [4,7] with mean $\left\langle v^{0}\right\rangle=1.34 \mathrm{~ms}^{-1}$ and standard deviation $\sqrt{\theta}=0.26 \mathrm{~ms}^{-1}$ 33. Speeds were limited to $v_{\alpha}^{\max }=1.3 v_{\alpha}^{0}$. Pedestrians enter the walkway at the ends at random positions. Those intending to walk from the left to the right hand side are represented by full circles, whereas pedestrians intending to move into the opposite direction are represented by empty circles. The diameter of a circle is a measure for the actual speed $\left\|\vec{v}_{\alpha}\right\|$ of a pedestrian $\alpha$. For

simplicity, no attractive effects $\vec{f}_{\alpha i}$ or fluctuations were taken into account. The repulsive potentials were assumed to decrease exponentially, i.e.

$$
V_{\alpha \beta}(b)=V_{\alpha \beta}^{0} \mathrm{e}^{-b / \sigma}, \quad U_{\alpha B}\left(\left\|\vec{r}_{\alpha B}\right\|\right)=U_{\alpha B}^{0} \mathrm{e}^{-\left\|\vec{r}_{\alpha B}\right\| / R}
$$


with $V_{\alpha \beta}^{0}=2.1 \mathrm{~m}^{2} \mathrm{~s}^{-2}, \sigma=0.3 \mathrm{~m}$ and $U_{\alpha B}^{0}=10 \mathrm{~m}^{2} \mathrm{~s}^{-2}, R=0.2 \mathrm{~m}$. Potentials with a hard core would be more realistic, of course, but they will not yield other results since the simulated pedestrians always keep enough distance.

Walkways were chosen $10 \mathrm{~m}$ wide. For $\Delta t$ in formula (4) we took $\Delta t=2 \mathrm{~s}$, and for the relaxation times we used $\tau_{\alpha}=0.5 \mathrm{~s}$. Smaller values of $\tau_{\alpha}$ let the pedestrians walk more aggressive. Finally, the effective angle of sight (which also takes into account head movements) was set to $2 \varphi=200^{\circ}$. Situations outside the angle of sight were assumed to have an influence of $c=0.5$. The model parameters introduced above were chosen in a way that is compatible with empirical data.

Figure 2 shows the empirically confirmed [6] development of dynamically varying lanes consisting of pedestrians who intend to walk into the same direction. Periodic boundary conditions in transversal direction would stabilize these lanes since they were not any more destroyed at the ends of the walkway by randomly entering pedestrians [2,21]. Figure 3 shows the number of forming lanes in dependence of the width of the walkway for a pedestrian density of $0.3 \mathrm{~m}^{-2}$.

The segregation effect of lane formation is not a result of the initial pedestrian configuration but a consequence of the pedestrians' interactions. Nevertheless, it normally leads to a more effective pedestrian flow since time-consuming avoidance maneuvers occur less frequently.

Figures 4 and 5 depict different moments of two pedestrian groups that try to pass a narrow door into opposite directions. The corresponding simulation shows the following: Once a pedestrian has passed the door, other pedestrians intending to move into the same direction are able to follow him/her easily (see Figure 4). However, the stream of passing pedestrians is stopped by the pressure of the opposing group after some time. Subsequently, the door is captured by pedestrians who pass the door into the opposite direction (see Figure 5). This change of the passing direction may occur several times and is well-known from observations. 


\section{SUMMARY AND OUTLOOK}

It has been shown that pedestrian motion can be described by a simple social force model for individual pedestrian behavior. Computer simulations of pedestrian groups demonstrated 1. the development of lanes consisting of pedestrians who walk into the same direction, 2 . oscillatory changes of the walking direction at narrow passages. These spatio-temporal patterns arise due to the nonlinear interactions of pedestrians. They are not the effect of strategical considerations of the individual pedestrians since they were assumed to behave in a rather 'automatical' way.

Presently, the social force model is extended by a model for the route choice behavior of pedestrians. As soon as the computer program is completed it will provide a comfortable tool for town- and traffic-planning.

The investigation of pedestrian behavior is an ideal starting point for the development

of other or more general quantitative behavioral models, since the variables of pedestrian motion are easily measurable so that corresponding models are comparable with empirical data. A further step could be the application of the social force concept to the description of opinion formation, group dynamics, or other social phenomena [26]. For this purpose, an abstract behavioral space has to be introduced.

\section{ACKNOWLEDGEMENTS}

The authors want to thank W. Weidlich, M. R. Schroeder, and W. Scholl for valuable and inspiring discussions. 


\section{REFERENCES}

[1] D. Helbing, Complex Systems 6, 391 (1992).

[2] D. Helbing, Physikalische Modellierung des dynamischen Verhaltens von Fußgängern (Diplom thesis, Georg-August University, Göttingen, Germany, 1990).

[3] D. Helbing, Stochastische Methoden, nichtlineare Dynamik und quantitative Modelle sozialer Prozesse (Shaker, Aachen, Germany, 1993).

[4] L. F. Henderson, Nature 229, 381 (1971).

[5] L. F. Henderson, Transp. Res. 8, 509 (1974).

[6] D. Oeding, Verkehrsbelastung und Dimensionierung von Gehwegen und anderen Anlagen des Fußgängerverkehrs (Straßenbau und Straßenverkehrstechnik, Heft 22, Bonn, Germany, 1963).

[7] F. P. D. Navin and R. J. Wheeler, Traffic Engineering 39, 30 (1969).

[8] Highway Capacity Manual, Chap. 13 (Transportation Research Board, Special Report 209, Washington, D.C., 1985).

[9] G. P. Gipps and B. Marksjö, Math. Comput. Simul. 27, 95 (1985).

[10] A. Borgers and H. Timmermans, Geographical Analysis 18, 115 (1986).

[11] G. G. Løvås, in Proceedings of the 1993 European Simulation Multiconference, Lyon, France, June 7-9, 1993.

[12] I. Prigogine and R. Herman, Kinetic Theory of Vehicular Traffic (American Elsevier, New York, 1971).

[13] S. L. Paveri-Fontana, Transp. Res. 9, 225 (1975).

[14] B. S. Kerner and P. Konhäuser, Phys. Rev. E 48, 2335 (1993).

[15] B. S. Kerner and P. Konhäuser, Phys. Rev. E 50, 54 (1994). 
[16] D. Helbing, An improved fluid-dynamic model for vehicular traffic, Phys. Rev. E. (submitted).

[17] O. Biham, A. A. Middleton, and D. Levine, Phys. Rev. A 46, 6124 (1992).

[18] K. Nagel and M. Schreckenberg, J. Phys. I France 2, 2221 (1992).

[19] T. Nagatani, Phys. Rev. E 48, 3290 (1993).

[20] J. A. Cuesta, F. C. Martínez, J. M. Molera, and A. Sánchez, Phys. Rev. E. 48, 4175 (1993).

[21] D. Helbing, Behavioral Science 36, 298 (1991).

[22] D. Helbing, in Natural Structures. Principles, Strategies, and Models in Architecture and Nature, Part II (Sonderforschungsbereich 230, Stuttgart, Germany, 1992).

[23] W. Weidlich and G. Haag, Concepts and Models of a Quantitative Sociology (Springer, Berlin, 1983).

[24] W. Weidlich, Physics Reports 204, 1 (1991).

[25] K. Lewin, Field Theory in Social Science (Harper \& Brothers, New York, 1951).

[26] D. Helbing, Physica A 196, 546 (1993).

[27] A. E. Scheflen and N. Ashcraft, Human Territories: How We Behave in Space-Time (Prentice-Hall, Englewood Cliffs, 1976).

[28] R. D. Freimuth and L. Lam, in Modeling Complex Phenomena, edited by L. Lam and V. Naroditsky (Springer, New York, 1992).

[29] D. R. Kayser, L. K. Aberle, R. D. Pochy, and L. Lam, Physica A 191, 17 (1992).

[30] F. Schweitzer and L. Schimansky-Geier, Physica A 206, 359 (1994).

[31] D. Helbing, P. Molnár, and F. Schweitzer, in Evolution of Natural Structures (Sonder- 
forschungsbereich 230, Stuttgart, Germany, 1994).

[32] F. Schweitzer, K. Lao, and F. Family, Active Walker simulate trunk trail formation by ants, Adaptive Behavior (submitted).

[33] U. Weidmann Transporttechnik der Fußgänger, pp. 87-88 (Schriftenreihe des Instituts für Verkehrsplanung, Transporttechnik, Straßen- und Eisenbahnbau Nr. 90, ETH Zürich, 1993). 


\section{TABLES}

\begin{tabular}{lll}
\hline \hline Stimulus & Simple/Standard & Complex/New \\
& Situations & Situations \\
Reaction & Automatic Re- & Result of Evaluation, \\
& action, 'Reflex' & Decision Process \\
Characterization & Well Predictable & Probabilistic \\
Modeling & Social Force & Decision Theore- \\
Concept & Model, etc. & tical Model, etc. \\
Example & Pedestrian & Destination Choice \\
& Motion & by Pedestrians \\
\hline \hline
\end{tabular}

TABLE I. Classification of behaviors according to their complexity. 


\section{FIGURES}

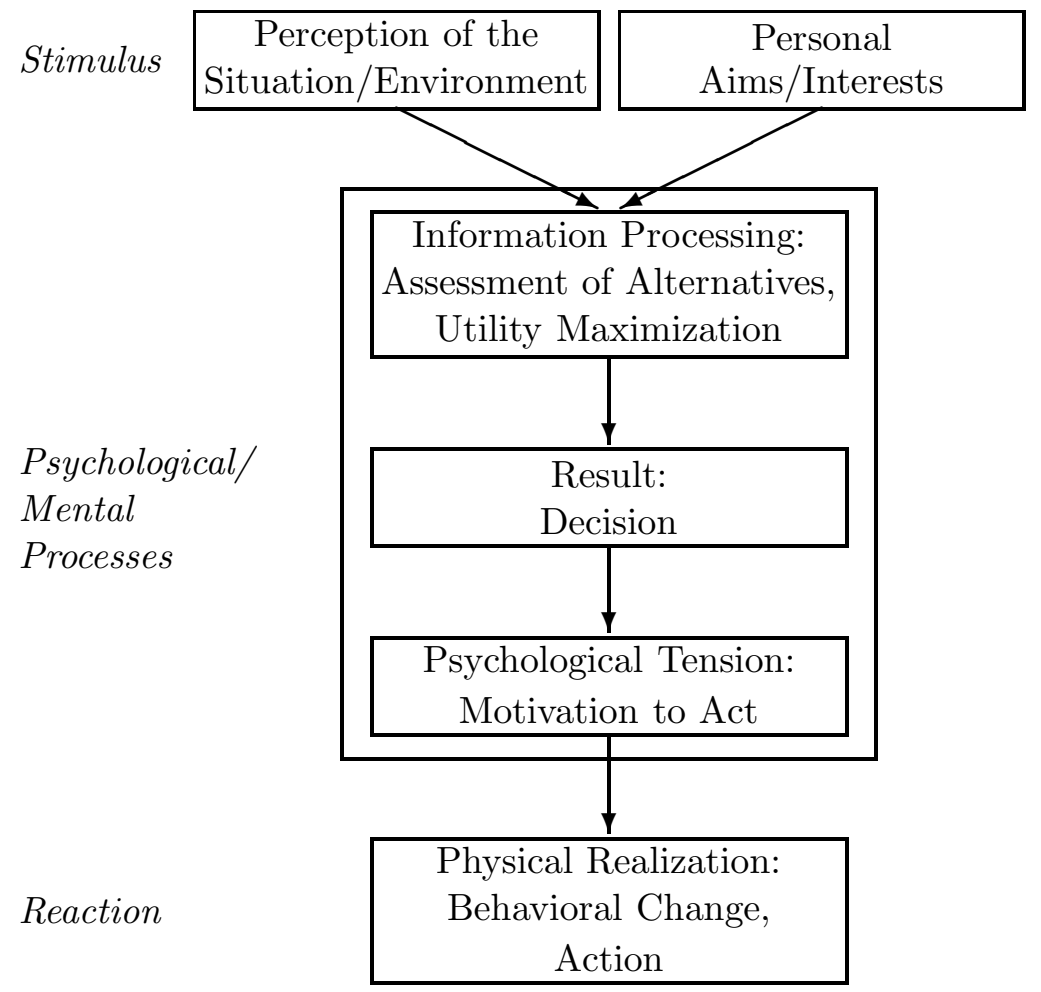

FIG. 1. Schematic representation of processes leading to behavioral changes. 
FIG. 2.

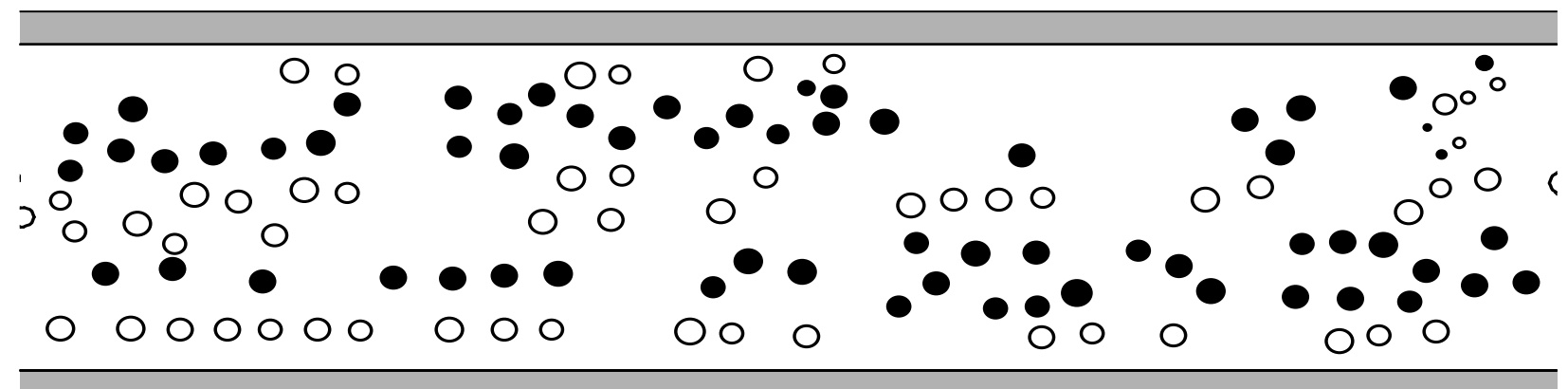

FIG. 2. Above a critical pedestrian density one can observe the formation of lanes consisting of pedestrians with a uniform walking direction. Here, the computational result shows $N=4$ lanes on a walkway that is $10 \mathrm{~m}$ wide and $50 \mathrm{~m}$ long. Empty circles represent pedestrians with a desired direction of motion which is opposite to that of pedestrians symbolized by full circles. 


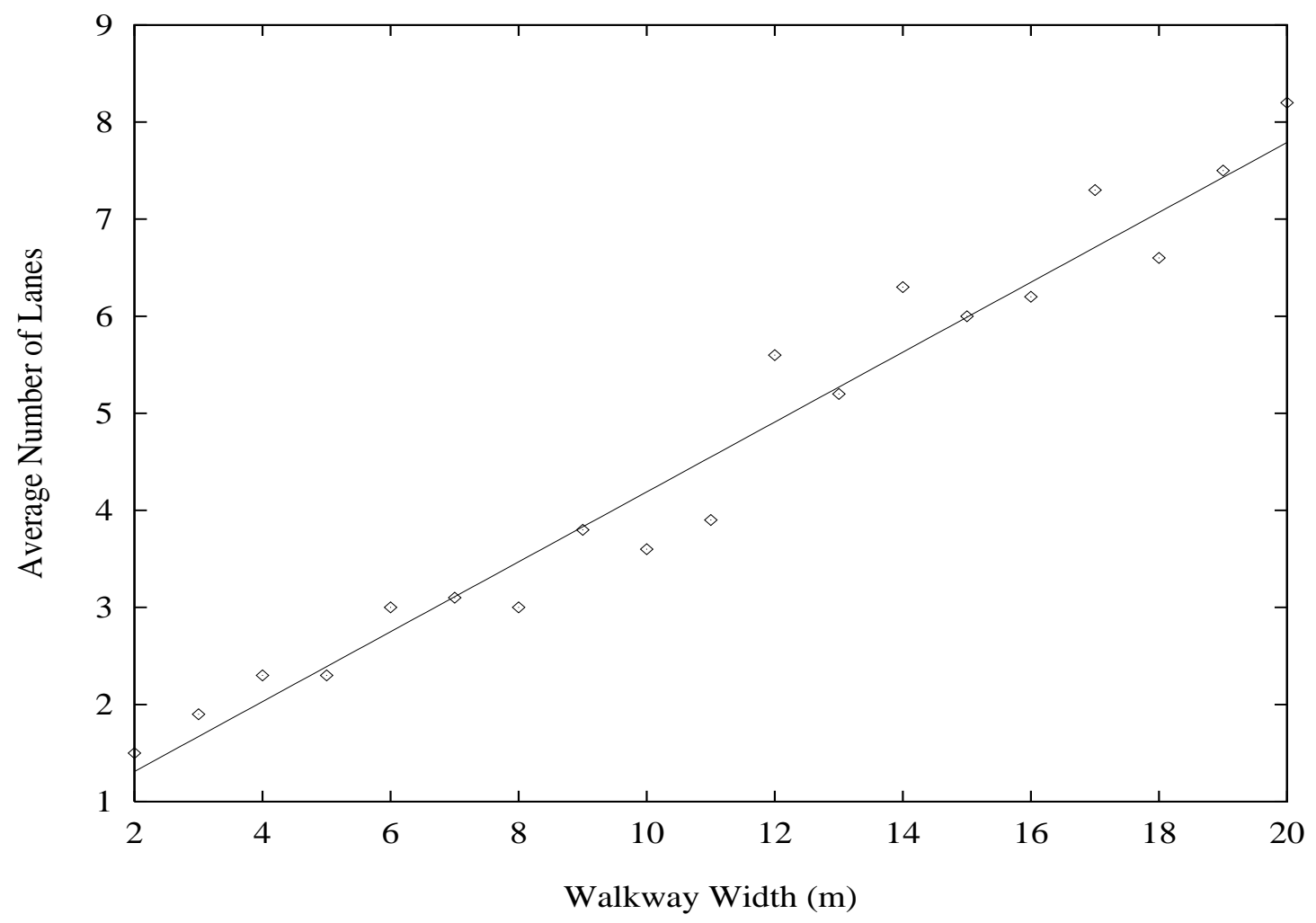

FIG. 3. The average number $N$ of lanes emerging on a walkway scales linearly with its width $W\left(N(W)=0.36 \mathrm{~m}^{-1} W+0.59\right)$. 
FIG. 4.

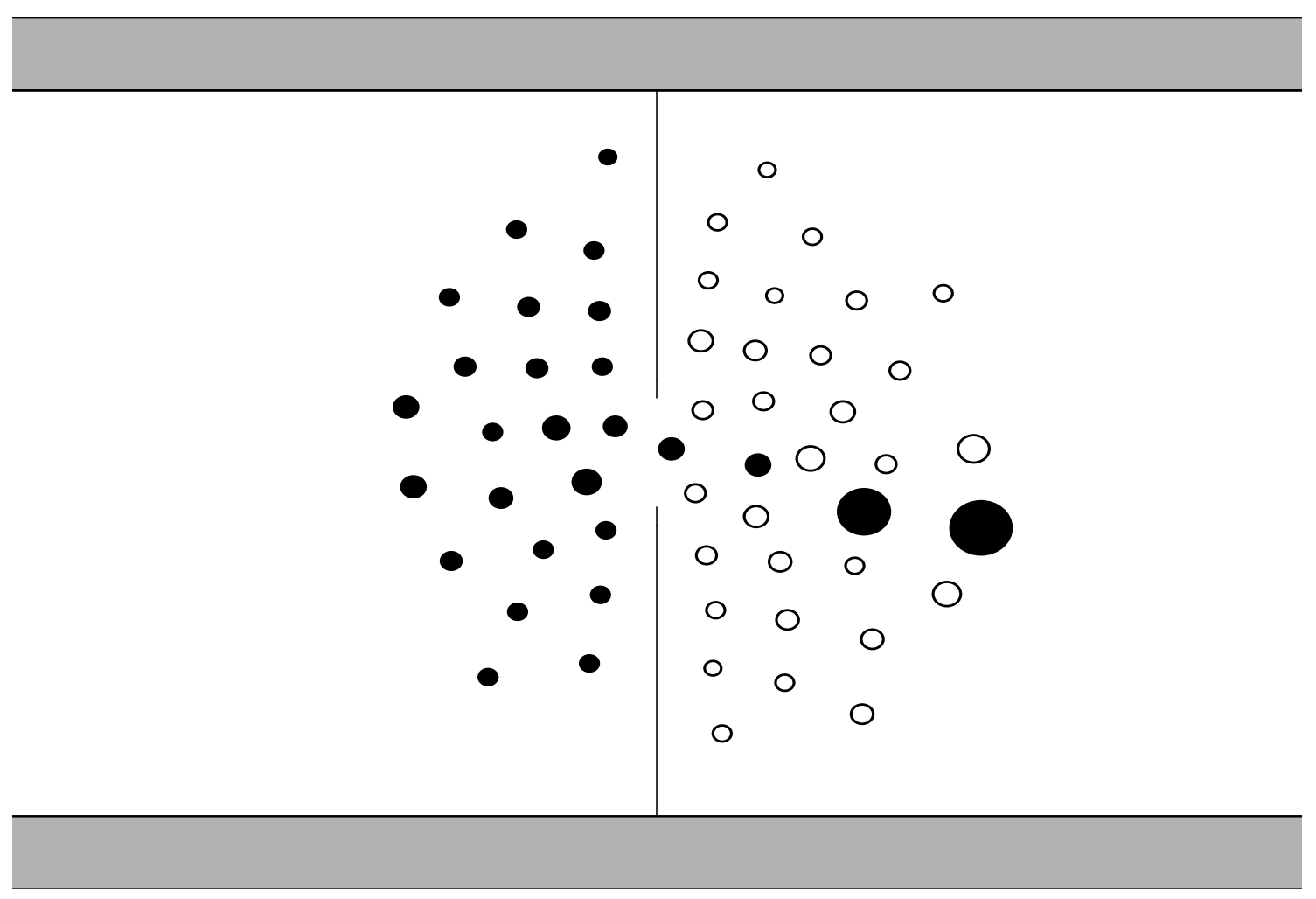

FIG. 4. If one pedestrian has been able to pass a narrow door, other pedestrians with the same desired walking direction can follow easily whereas pedestrians with an opposite desired direction of motion have to wait. The diameters of the circles are a measure for the actual velocity of motion. 
FIG. 5.

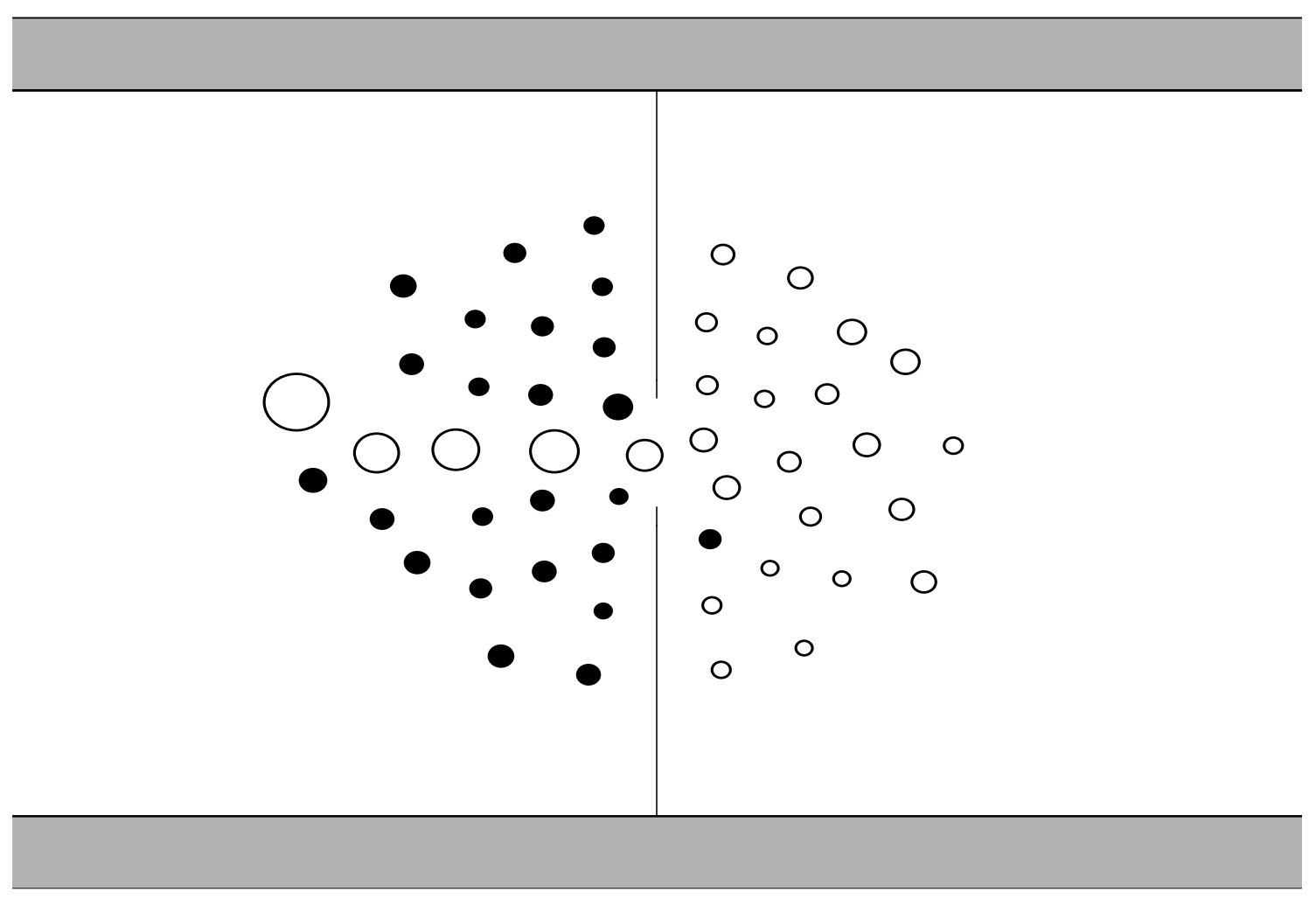

FIG. 5. After some time the pedestrian stream is stopped, and the door is captured by individuals who pass the door into the opposite direction. 